\title{
III. Evaluación clínica de los pacientes con enfermedad pulmonar obstructiva crónica en los programas de rehabilitación respiratoria
}

\author{
JUANA PAVIÉ G.*, MANUEL DE LA PRIDA C.*, FRANCISCO ARANCIBIA H.**,***, \\ CAROLA VALENCIA S.*,****, MARÍA JOSÉ HERRERA R.*,****, \\ SUSANA JORCANO S.*,****, XIMENA VALLE M.*,****, NORMA SARMIENTO CH.*,****, \\ KAREN STÖRM V.*,**** y ERICK LETELIER M.*,****
}

Clinical evaluation of patients with chronic obstructive pulmonary disease in pulmonary rehabilitation programs

Patients with Chronic Obstructive Pulmonary Disease (COPD) are the largest tributaries of the pulmonary rehabilitation programs. This chapter discusses the necessary evaluation required for patients with COPD, before entering the pulmonary rehabilitation program. Scientific evidence exists regarding the benefits of these programs. The assessment method recommend is: general evaluation, lung function, exercise tolerance (6-minute walk test, incremental walking test), dyspnea (scale of Borg, modified Medical Research Council scale) and health-related quality of life (Saint George's questionnaire). Also, BODE index, psychological and nutritional assessment and a cardiovascular evaluation to rule out cardiac pathology that contraindicates rehabilitation, should be carried out. Following the evaluation, the patient will be into a pulmonary rehabilitation program, the team should consist of a multidisciplinary and include: 1) education of patients and their families, 2) muscle testing and training of: lower extremities, upper extremities and respiratory muscles, and 4) psychosocial support. The pulmonary rehabilitation program provides significant benefits to patients with COPD in terms of reducing dyspnea, improve exercise capacity and quality of life (quality evidence A, strong recommendation). Physical space is required for the evaluation of patients and a training room. It is recommended that pulmonary rehabilitation program must be personalized and centred on the needs of the patient and has a duration of 6 to 12 weeks. Programs effectiveness is independent of where they are carried out and it depends primarily on its structure. It is important to work out a strategy study and control program for evaluating its success.

Key words: Pulmonary rehabilitation program, assessment method, COPD.

\section{Resumen}

Los pacientes con Enfermedad Pulmonar Obstructiva crónica (EPOC) son los mayores tributarios de los programas de rehabilitación respiratoria. En este capitulo se analiza la evaluación que requieren los pacientes con EPOC antes de ingresar al programa de rehabilitación respiratoria y la evidencia científica que existe en cuanto a sus beneficios. El método de evaluación recomendado es: una evaluación general, la función pulmonar, la tolerancia al ejercicio (prueba de caminata de 6 minutos, prueba de caminata incremental), la disnea (escala de Borg, escala del Medical Research Council modificada) y los relacionados con la calidad de vida con el cuestionario de Saint George. Además, evaluación del índice BODE, evaluación psicológica y nutricional y una evaluación cardiovascular para descartar patología cardiaca que contraindique la rehabilitación. Efectuada la evaluación, se ingresa al paciente a un programa de rehabilitación respiratoria, el cual debe constar de un equipo multidisciplinario y debe incluir : 1) Educación de los pacientes y su familia; 2) Evaluación y entrenamiento muscular de extremidades inferiores, superiores y músculos respiratorios; 3) Soporte nutricional, y 4) Apoyo psicosocial. El programa de rehabilitación pulmonar proporciona importantes beneficios a los pacientes

\footnotetext{
* $\quad$ Servicio de Salud Viña del Mar - Quillota, V Región.

** Servicio Médico Respiratorio y Unidad de Paciente Crítico, Instituto Nacional del Tórax.

*** Unidad de Respiratorio, Clínica Santa María.

**** Kinesiólogo (a).
} 
con EPOC en términos de reducción de la disnea, mejoría en la capacidad de ejercicio y en la calidad de vida (calidad de la evidencia A, recomendación fuerte). Se debe contar con espacio físico para la evaluación de los pacientes y con una sala de entrenamiento. Se recomienda que el programa de rehabilitación respiratoria debe ser personalizado y centrado en las necesidades del paciente y debe tener una duración de 6 a 12 semanas. La efectividad del programa es independiente del lugar donde se lleva a cabo y depende principalmente de su estructura. Es importante elaborar un estudio de estrategia y programa de control para evaluar su éxito.

Palabras clave: Programas de rehabilitación respiratoria, método de evaluación, EPOC.

\section{Introducción}

El paciente con Enfermedad Pulmonar Obstructiva Crónica (EPOC) es el mayor tributario de los programas de rehabilitación respiratoria (RR) en todo el mundo ${ }^{1}$ y esto es lógico si se considera que esta enfermedad continúa aumentado tanto en su prevalencia como en su mortalidad. Se estima que esta entidad clínica será la tercera causa de muerte en la población mundial durante el próximo decenio ${ }^{2}$. Si bien es cierto que el abandono del hábito tabáquico constituye la principal medida costo-efectiva en el manejo global de la enfermedad, la efectividad de los programas de RR como intervención integrativa en la EPOC ha sido demostrada ampliamente ${ }^{1-3}$. Esto no implica que la rehabilitación no se pueda aplicar en las otras enfermedades respiratorias crónicas, sobre todo considerando que la mayor parte de ellas también tiene un componente multisistémico, con morbilidades secundarias ${ }^{7}$, además del compromiso respiratorio de la EPOC y de hecho así lo demuestra la literatura ${ }^{4-6}$. Aún así, es en el paciente con EPOC donde con mayor frecuencia se realiza esta intervención y donde la mayoría de la evidencia ha demostrado la utilidad de los programas de rehabilitación respiratoria ${ }^{8}$.

Los programas de rehabilitación respiratoria se definen como una "intervención multidisciplinaria basada en la evidencia en pacientes portadores de enfermedades respiratorias crónicas, que son sintomáticos y con disminución de la capacidad física, integrada al tratamiento individualizado del paciente, con el propósito de reducir los síntomas, mejorar la capacidad funcional, aumentar la participación y reducir los costos asociados al cuidado de la salud, estabilizando o revirtiendo las manifestaciones sistémicas de la enfermedad $^{1,9}$. Se debe recomendar los programas de rehabilitación respiratoria a todo paciente con EPOC que persiste sintomático después de recibir un tratamiento medicamentoso y no medicamentoso apropiado ${ }^{10}$.

Los objetivos básicos de la rehabilitación respiratoria en el paciente con EPOC son reducir la disnea, aumentar la tolerancia al ejercicio y mejorar la calidad de vida. Aspectos que están comprometidos en esta enfermedad en diferentes grados dependiendo de la etapa evolutiva en que se encuentra ${ }^{1,9,11}$. Para alcanzar estos objetivos, la rehabilitación pulmonar debe cubrir un amplio espectro de problemas pulmonares y extrapulmonares, que no son adecuadamente abordados por el tratamiento medicamentoso, tales como la falta de entrenamiento físico, el relativo aislamiento social, la alteración del estado de ánimo (en especial la depresión), la pérdida de masa muscular y la reducción de peso corporal ${ }^{12}$.

No se debe considerar a la rehabilitación respiratoria como el último recurso en los pacientes con EPOC avanzado, y si nos atenemos a la clasificación GOLD ${ }^{13}$, se ha demostrado la utilidad de la RR desde los estadios moderados (GOLD II) hacia delante incluyendo los casos muy severos, independiente de la edad, la presencia de hipoxemia y/o hipercapnia y la presencia o no de tabaquismo ${ }^{1,9,13}$. En todos ellos, los programas de RR han mejorado uno o más de los aspectos considerados en los objetivos detallados previamente, lo cual puede variar dependiendo del estado de la enfermedad ${ }^{14}$. En este sentido es de vital importancia determinar cuáles son los métodos de evaluación que nos permitirán pesquisar a aquellos pacientes con EPOC que se beneficiarán con esta intervención, definiendo su gravedad inicial, magnitud de la disnea como síntoma importante en estos pacientes, la tolerancia al ejercicio y objetivar la calidad de vida. También la evaluación clínica deberá estar orientada a determinar eventuales criterios de exclusión que puedan contraindicar esta intervención, así como para disponer de parámetros de evaluación útiles para el seguimiento de los enfermos dentro del programa y una vez que éste haya finalizado ${ }^{1,3,9,11,13}$.

\section{Preguntas}

1. ¿Los programas de rehabilitación respiratoria son beneficiosos para el paciente con EPOC?

2. ¿Qué pacientes debieran ingresar a un programa de rehabilitación respiratoria y cuáles son los criterios de exclusión?

3. ¿Qué metodología de evaluación se recomienda en pacientes con EPOC que ingresan a un programa de rehabilitación respiratoria? 


\section{Pacientes}

Pacientes portadores de EPOC desde un estadio GOLD II en adelante, que no presenten criterios de exclusión.

\section{Intervención}

Metodología de evaluación clínica y funcional en pacientes con EPOC que ingresan a un programa de rehabilitación respiratoria.

\section{Objetivo}

Examinar la metodología de evaluación clínica $\mathrm{y}$ funcional de los pacientes con EPOC que ingresan a un programa de rehabilitación respiratoria, que nos permita discriminar a los pacientes que se beneficiarán de la intervención, clasificarlos de acuerdo a su severidad y diferenciar los que presenten criterios de exclusión de acuerdo a la literatura.

\section{Resumen de la evidencia}

En 1996, Lacasse y cols, publicaron un meta-análisis que examinaba la eficacia de los programas de rehabilitación respiratoria en pacientes con EPOC y evaluaron el efecto sobre los síntomas, la capacidad de ejercicio y la calidad de vida. Los autores revisaron sistemáticamente catorce estudios aleatorizados y controlados que evaluaron programas de entrenamiento físico de al menos cuatro semanas de duración asociado o no a educación y/o soporte psicológico. Los grupos control no realizaron rehabilitación respiratoria. Ellos concluyen que los pacientes con EPOC que recibieron rehabilitación respiratoria presentaron mejoría significativa de la disnea, capacidad de ejercicio (media: 55,7 m), capacidad de esfuerzo máximo (media: 8,3 W) y calidad de vida relacionado a la salud. Estas mejorías son clínicamente importantes y después de este análisis quedó claro que los programas de rehabilitación respiratoria deberían constituir una parte integral de la atención de los pacientes con $\mathrm{EPOC}^{15}$.

Posteriormente, estos datos fueron confirmados por otro estudio publicado el año 2006 por la base de datos Cochrane, realizado sobre 31 estudios aleatorizados controlados. Ellos concluyen que la rehabilitación respiratoria ha demostrado ampliamente su eficacia en el paciente con EPOC y no se requieren más estudios que validen su utilidad 9 .

También existe evidencia que los pacientes con EPOC que participan en un programa de rehabilitación respiratoria tienen impacto en la utilización de los recursos de salud. Griffiths y cols ${ }^{16}$, publicaron un estudio que incluía a 200 pacientes, los cuales fueron asignados al azar a un programa multidisciplinario de RR durante seis semanas $(\mathrm{n}=99)$ o manejo médico estándar (n $=101$ ), y se evaluó el uso de servicios de salud. Los autores no encontraron diferencias significativas en la admisiones hospitalarias, pero si en el tiempo de estadía hospitalaria siendo menor en el grupo que realizó $\mathrm{RR}(10,4 \pm 9,7$ días vs $21,0 \pm$ $20,7, \mathrm{p}=0,022)$. Además, los pacientes rehabilitados requirieron menor números de visitas domiciliarias de los servicios de atención primaria $(1,5 \pm 2,8$ vs $2,8 \pm 4,6, p=0,037)$ en comparación con el grupo control. En otro estudio, Puman y $\operatorname{cols}^{17}$, encontraron que la RR, después de una exacerbación aguda, permitía reducir significativamente el riesgo de rehospitalización. Además, se ha demostrado que la rehabilitación respiratoria realizada en forma precoz en el período de recuperación después del alta hospitalaria de una exacerbación aguda de la EPOC, conduce a mejorías significativas en la capacidad funcional y calidad de vida de los enfermos comparado con la atención habitual ${ }^{18}$.

Además, se ha comunicado que los pacientes con EPOC que participan en un programa de RR pueden mejorar el índice BODE. En un estudio prospectivo de caso-control de tres años de duración en que fueron incluidos 80 pacientes con EPOC moderado a severo, se encontró que la rehabilitación respiratoria comparada con el tratamiento usual mejoraba el índice de masa corporal (IMC), la función pulmonar y el estado de salud ${ }^{19}$. Otros autores también han encontrado mejoría del índice BODE con esta intervención ${ }^{20}$. De este modo, los programas de RR deberían estar disponibles para todos los pacientes con EPOC moderado, grave o muy grave y/o que se consideren ellos mismos con algún grado de incapacidad ${ }^{9111,21,22}$.

No se recomienda esta intervención a los pacientes que presenten alguna disfunción del aparato locomotor que les impida la realización de ejercicios o del sistema cardiovascular como angina inestable, infarto agudo de miocardio reciente, estenosis aórtica severa, insuficiencia cardíaca grave refractaria a tratamiento, arritmias cardíacas graves o hipertensión pulmonar seve$\mathrm{ra}^{9,13,23}$. En la Tabla 1 se enumeran las principales entidades clínicas que limitan el ingreso a un programa de rehabilitación respiratoria.

La mayor parte de los trabajos, como los de Sabit y $\operatorname{cols}^{24}$ y Haave y $\operatorname{cols}^{25}$, demuestran que los factores como la edad, género y la condición de fumador no excluyen de los beneficios de la rehabilitación respiratoria, aún cuando se ha demostrado que pueden determinar junto a los 
Tabla 1. Contraindicaciones de los pacientes con EPOC para ingresar a un programa de rehabilitación respiratoria

\begin{tabular}{|l|}
\hline Pobre motivación o falta de colaboración \\
Trastornos psiquiátricos severos no compensados \\
Estenosis aórtica \\
Arritmias: Fibrilación auricular-Extrasistolía ventri- \\
cular frecuente \\
Insuficiencia cardíaca CF III-IV \\
Angina inestable o a esfuerzos leves \\
Infarto agudo de miocardio reciente (menos de 3 \\
meses) \\
Aneurisma aórtico o cerebral \\
Bloqueo aurículo-ventricular completo \\
Hipertensión arterial descompensada (PAD $\geq 100$ \\
mmHg y/o PAS $\geq 180$ mmHg) \\
PAD mayor o igual a 115 mmHg durante el ejercicio \\
Hipertensión pulmonar severa \\
Trombosis venosa profunda reciente (menor a 1 mes) \\
Tromboembolismo pulmonar o accidente vascular \\
cerebral reciente (menor a 1 mes) \\
Aterosclerosis de extremidades inferiores severa: clau- \\
dicación intermitente \\
Frecuencia cardíaca en reposos mayor o igual a 120 \\
ciclos/min \\
Infección aguda (ej: Neumonía comunitaria) \\
Diabetes mellitus no controlada \\
Tirotoxicosis - Mixedema \\
Insuficiencia renal \\
Hipokalemia \\
Exacerbación de la enfermedad respiratoria de base al \\
momento de la evaluación \\
Alteraciones neuro-músculo-esqueléticas \\
Enfermedades del aparato locomotor, accidentes \\
colaboración en el programa de entrenamiento
\end{tabular}

PAD: presión arterial diastólica; PAS: presión arterial sistólica.

factores psicosociales menor adherencia o incumplimiento de esta terapia no farmacológica ${ }^{26,27}$.

Un paciente con EPOC, candidato a ingresar a un programa de rehabilitación respiratoria debe contar en primer lugar con una historia médica completa, incluyendo los antecedentes personales, hábitos con especial énfasis en tabaquismo, antecedentes laborales, entorno y soporte familiar y social, grado de motivación y la presencia de comorbilidades no respiratorias, así como el examen físico correspondiente, también debe solicitarse una evaluación básica de laboratorio que incluya hemograma, parámetros bioquímicos, gases en sangre arterial y electrolitos plasmáticos ${ }^{13}$.

De igual modo, todos los pacientes deben contar, para establecer el diagnóstico y la severidad de la EPOC, con una espirometría con y sin broncodilatador ${ }^{13,28,29}$. En cuanto a los exámenes complementarios, podemos diferenciar aquellos que evalúan la magnitud de la disnea, la capacidad de ejercicio, calidad de vida, fuerza muscular inspiratoria y espiratoria, fuerza muscular de extremidades inferiores y superiores, evaluación global con índice BODE, evaluación psicológica, evaluación nutricional y evaluación cardiovascular.

\section{Evaluación de la capacidad al ejercicio}

a) Prueba de caminata de 6 minutos. Ésta nos permite objetivar por una parte la magnitud de la disnea y como lo demuestran Spencer y $\operatorname{cols}^{30}$, el efecto de la RR en la capacidad de realizar ejercicio durante la rehabilitación y después de ella ${ }^{31-33}$. En los programas de rehabilitación pulmonar se espera obtener un rango de incremento de 25 a 54 m ó $10 \%$ sobre el examen basal ${ }^{34,35}$.

b) Prueba de caminata incremental ${ }^{36}$. Se emplea también para evaluar la capacidad de realizar ejercicio, específicamente puede ser usado para determinar la carga de entrenamiento inicial. Se estima que aumente con la RR de acuerdo a Singh y $\operatorname{cols}^{37}$, a lo menos 48 metros.

\section{Evaluación del impacto de la disnea y del estado de salud relacionado con la calidad de vida}

La evaluación de la disnea también es un componente fundamental dentro del programa, ya que ésta es un síntoma predominante y determinante de la limitación de la actividad física de los pacientes, siendo el más relevante por su naturaleza invalidante ${ }^{11,13,14}$. Además, existe una fuerte relación entre la disnea y las pruebas asociadas a actividades de la vida diaria, como la prueba de caminata de 6 minutos.

Existen diferentes tipos de escalas, que buscan valorar la disnea en diferentes aspectos o situaciones (durante el ejercicio, en actividades de la vida diaria o como condicionante de la calidad de vida). En cambio, el impacto social de la enfermedad respiratoria debe ser medido con instrumentos que evalúen el estado de salud, previamente referidos como medidas de calidad de vida. 
Tabla 2. Índice BODE para la evaluación de pacientes con enfermedad pulmonar obstructiva crónica

\begin{tabular}{|lcccc|}
\hline Variable & \multicolumn{4}{c}{ Puntos en índice BODE } \\
\hline VEF $_{1}$ (porcentaje teórico) & $\mathbf{0}$ & $\mathbf{1}$ & $\mathbf{2}$ & $\mathbf{3}$ \\
\hline Distancia caminada 6 minutos $(\mathrm{m})$ & $\geq \mathrm{a} 65$ & $50-64$ & $36-49$ & $\leq 35$ \\
MMRC escala de disnea & $\geq 350$ & $250-349$ & $150-249$ & $\leq 149$ \\
\hline Índice de masa corporal $\left(\mathrm{kg} / \mathrm{m}^{2}\right)$ & $0-1$ & 2 & 3 & 4 \\
\hline
\end{tabular}

a) Escala de Borg de disnea durante el ejercicio o escala visual análoga. Se utilizará sólo durante la prueba de caminata, como lo señala la norma técnica de este examen, ya que es una escala de evaluación de la disnea aguda o relacionada a actividad específica ${ }^{38,39}$.

b) Escala de disnea del Medical Research Council Modificada (MMRC). Esta escala emplea un rango de puntuación entre 0 (sin disnea) y 4 (máxima disnea) ${ }^{40}$. Esta escala ha demostrado ser más útil para discriminar entre pacientes que para evaluar los efectos de la $\mathrm{RR}^{41}$.

La evaluación del estado de salud implica una cuantificación estandarizada del impacto de la enfermedad y es uno de los principales resultados valorados en los programas de rehabilitación ${ }^{1,9,11}$. Indirectamente, se evalúa a través de cuestionarios de calidad de vida, de los cuales el más usado en los programas de rehabilitación respiratoria ha sido el Cuestionario Respiratorio de Saint George (SGRQ) del Dr. Paul Jones (1991). El cuestionario evalúa tres componentes: los síntomas, las actividades y el impacto de la enfermedad, en 50 ítems ${ }^{29}$. Permite evaluar los efectos del tratamiento y las intervenciones de manejo en el paciente con EPOC. Se sugiere que este cuestionario sea aplicado por personal entrenado o capacitado en ello. En un programa de rehabilitación respiratoria se espera obtener una diferencia mayor o igual a 4 puntos para considerar que se logró un cambio significativo en la calidad de vida ${ }^{42}$.

Evaluación de la fuerza muscular inspiratoria y espiratoria y la fuerza muscular de las extremidades inferiores (cuádriceps), especialmente en los pacientes que presentan atrofia muscular

a) La medición de la fuerza de los músculos respiratorios se realiza mediante la medición de la Presión Inspiratoria Máxima (Pimax) y Presión Espiratoria Máxima (Pemax), que se logra mediante un manovacuómetro ${ }^{43,44}$.

b) Se recomienda evaluar la fuerza de las extremidades superiores e inferiores, determinando la carga de trabajo que puede soportar. Puede utilizarse mediciones circunferenciales o transversales o a través de imágenes (ecografía, tomografía o RNM) en casos específicos. Esto nos permite elegir el método de entrenamiento más recomendable $3,9,13,45$.

\section{Evaluación global de la EPOC: Índice BODE}

A pesar de haber sido desarrollado como un marcador pronóstico en los pacientes con EPOC especialmente en lo referente a mortalidad $^{46}$, el índice BODE últimamente ha ido ganado más aceptación como método evaluativo en otros aspectos de la enfermedad, uno de ellos sería para medir la eficacia terapéutica de los programas de rehabilitación respiratoria ${ }^{20}$. El valor del índice BODE se obtiene a partir de puntajes asignados a cada una de las siguientes cuatro variables: 1) $\mathrm{VEF}_{1}$ (porcentaje del valor teórico post broncodilatador); 2) Prueba de caminata de 6 minutos (en metros); 3) Magnitud de la disnea por escala MMCR; y 4) Índice de masa corporal: peso corporal $/ \mathrm{talla}^{2}\left(\mathrm{~kg} / \mathrm{m}^{2}\right)$ (Tabla 2$)$.

Dado que se obtiene a partir de parámetros usualmente registrados en la evaluación de los pacientes con EPOC que ingresaran a un programa de RR, se aconseja su empleo, ya que su medición permite integrar diferentes aspectos de la EPOC y el riesgo asociado a comorbilidades significativas.

\section{Evaluación psicológica}

Es frecuente que los pacientes con EPOC avanzado presenten cuadros de ansiedad y depresión asociados a su patología de base ${ }^{47}$. $\mathrm{Si}$ bien, son escasos los estudios que han evaluado el efecto de la RR en los trastornos del ánimo de los enfermos, y un estudio realizado por Toshima y cols, $(n=119)$ no demostró efectos beneficiosos ${ }^{48}$; no obstante, se recomienda realizar una evaluación psicológica, en particular de aquellos con trastornos depresivos severos, antes de ingresar a un programa de RR. En nuestro medio 
Tabla 3. Programas de rehabilitación respiratoria y entrenamiento muscular recomendados por Sociedad Española de Neumología y Cirugía Torácica (SEPAR) y la Asociación Latinoamericana de Tórax (ALAT) ${ }^{10}$

\begin{tabular}{|c|c|c|}
\hline Programa & Técnicas & Requisitos \\
\hline $\begin{array}{l}\text { Entrenamiento a resistencia de extre- } \\
\text { midades inferiores }\end{array}$ & $\begin{array}{l}\text { - Bicicleta } \\
\text { - Tapiz rodante } \\
\text { - Andar } \\
\text { - Subir escaleras }\end{array}$ & $\begin{array}{l}\text { A una intensidad suficiente de su } \\
\text { capacidad máxima (evaluada por } \\
\text { consumo de } \mathrm{O}_{2} \text {, frecuencia cardíaca } \\
\text { o síntomas) } \\
\text { Duración: } 30-45 \mathrm{~min} / \text { día, tres veces } \\
\text { por semana }\end{array}$ \\
\hline $\begin{array}{l}\text { Entrenamiento a resistencia de extre- } \\
\text { midades superiores }\end{array}$ & $\begin{array}{l}\text { Movimientos sin soporte } \\
\text { (levantamiento de pesos } \\
\text { pequeños o barra) } \\
\text { Movimientos con soporte } \\
\text { (ergonómetro de brazos) }\end{array}$ & $\begin{array}{l}\text { Duración: } 30 \mathrm{~min} / \text { día, tres veces por } \\
\text { semana }\end{array}$ \\
\hline $\begin{array}{l}\text { Entrenamiento a fuerza de extremi- } \\
\text { dades }\end{array}$ & $\begin{array}{l}\text { - Ejercicio con pesas o } \\
\text { aparatos gimnásticos }\end{array}$ & $\begin{array}{l}\text { Cargas elevada (aproximadamente } \\
80 \% \text { máximo tolerado). Series de } \\
\text { pocas repeticiones }\end{array}$ \\
\hline $\begin{array}{l}\text { Entrenamiento de músculos respi- } \\
\text { ratorios }\end{array}$ & $\begin{array}{l}\text { - Umbral de presión }\left(\text { Threshold }^{\circledR}\right) \\
\text { - Resistencia }\left(\text { Plex }^{\circledR}\right)\end{array}$ & $\begin{array}{l}\text { Control del patrón respiratorio. In- } \\
\text { tensidad equivalente al } 30 \% \text { de la } \\
\text { presión inspiratoria máxima } \\
\text { Duración: } 15 \mathrm{~min} / \text { día, } 2 \text { sesiones/día }\end{array}$ \\
\hline
\end{tabular}

Todos los programas deben ser precedidos de maniobras de calentamiento y estiramiento.

podemos utilizar la escala de depresión CIE 10 (Ministerio de Salud de Chile) disponible en salud pública. A nivel internacional se emplea la escala hospitalaria de ansiedad y depresión de Zigmond $^{49}$, la cual está validada.

\section{Evaluación nutricional}

Es recomendable incluir en la evaluación de los candidatos a RR, un informe nutricional a cargo de especialista y consignar el índice de masa corporal (IMC) de cada paciente, dado el factor pronóstico que ello conlleva ${ }^{50,51}$, tratando de pesquisar a los pacientes con déficit de peso (desnutrición) y/o desbalance entre la masa magra y masa grasa ${ }^{52}$.

\section{Evaluación cardiovascular}

El objetivo básico es discriminar entre los pacientes que eventualmente pudiesen tener alguna comorbilidad cardiovascular que pudiera poner en peligro su vida al realizar el programa de entrenamiento muscular, en particular los diagnósticos de angina inestable y/o estenosis aórtica severa. La presencia de hipertensión pulmonar no contraindicaría la rehabilitación respiratoria, a menos que sea severa y se acompañe de síncope. En este sentido, se recomienda realizar un electrocardiograma de reposo a todos los pacientes como método de pesquisa y eventualmente una ecocardiografía bidimensional si se sospecha patología cardíaca ${ }^{3,7,9,11}$.

\section{Ficha de recolección de información}

Los pacientes que ingresen al programa es importante que mantengan en forma independiente, además de su ficha clínica, una hoja de recolección de datos que los identifique, contenga sus antecedentes clínicos básicos, los parámetros de evaluación realizados a su ingreso al programa de $\mathrm{RR}$, la clasificación diagnóstica, su severidad y la posibilidad de reevaluaciones en el tiempo. Esto permite definir el lugar y la modalidad de rehabilitación respiratoria para cada paciente y por otro lado, realizar evaluaciones periódicas del propio paciente y del programa en general ${ }^{53}$. La Figura 1 muestra un ejemplo de ficha de recolección de datos para un programa de rehabilitación respiratoria, la cual puede ser modificada de acuerdo a la realidad local de cada lugar.

\section{Componentes mínimos de un programa de rehabilitación respiratoria}

Se requiere de un equipo multidisciplinario, con el fin de aplicar el programa de rehabilitación respiratoria, el cual define y determina el personal necesario para realizarlo, el cual debe estar integrado por lo menos por un médico coordinador, idealmente un neumólogo encargado de evaluar a los enfermos, y un profesional de la salud (kinesiólogo) que efectúe las pruebas y realice el entrenamiento físico ${ }^{41}$. El programa de rehabilitación debe incluir los siguientes elementos fundamentales: 1) Educación de los pacientes y su 
familia; 2) Evaluación y entrenamiento muscular de extremidades inferiores, superiores y músculos respiratorios (Tabla 3); 3) Soporte nutricional, y 4) Apoyo psicosocial.
Estructura y equipamiento requerido para un programa de rehabilitación respiratoria

Para realizar un programa de rehabilitación respiratoria se requiere de espacio físico para

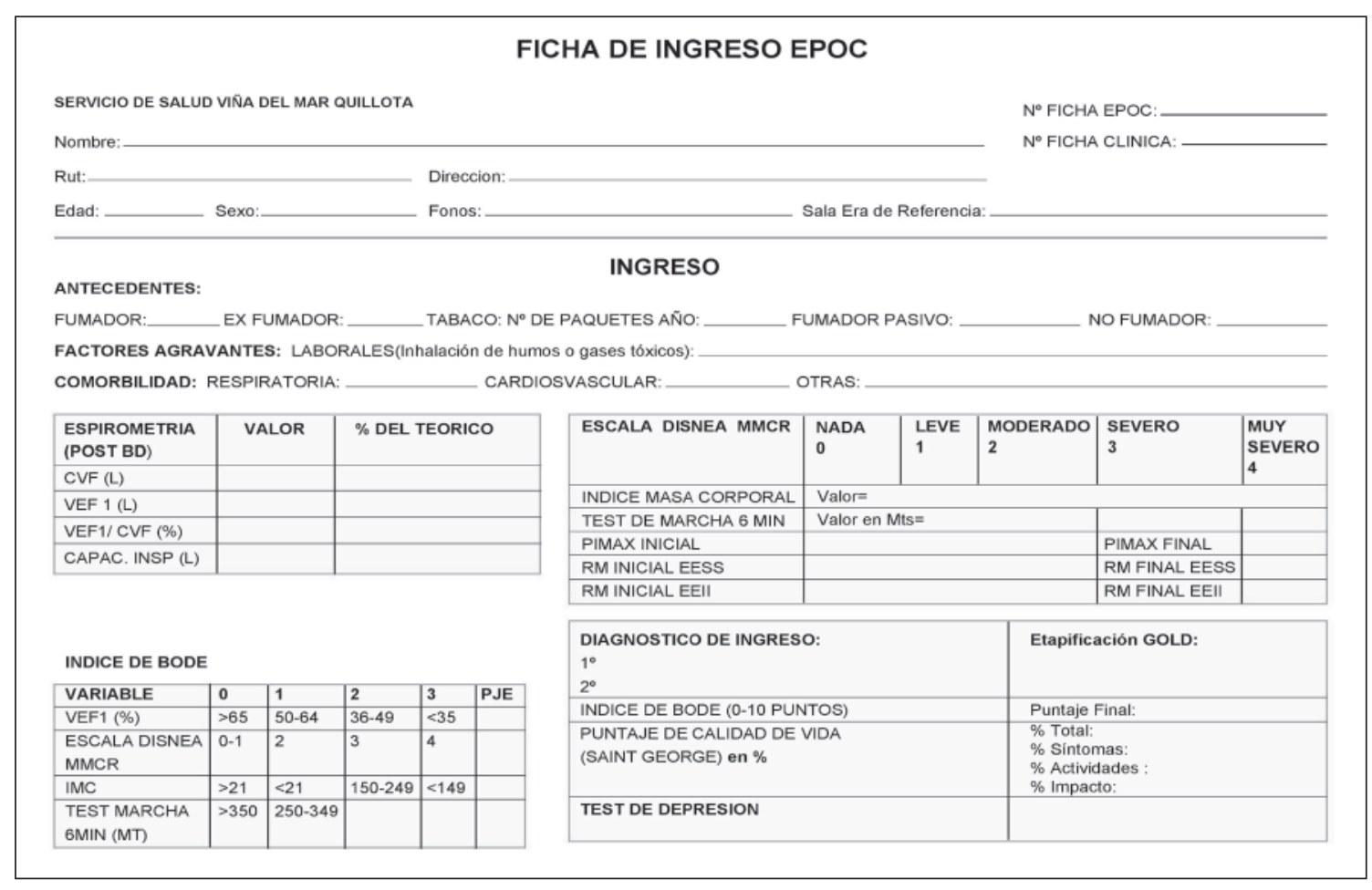

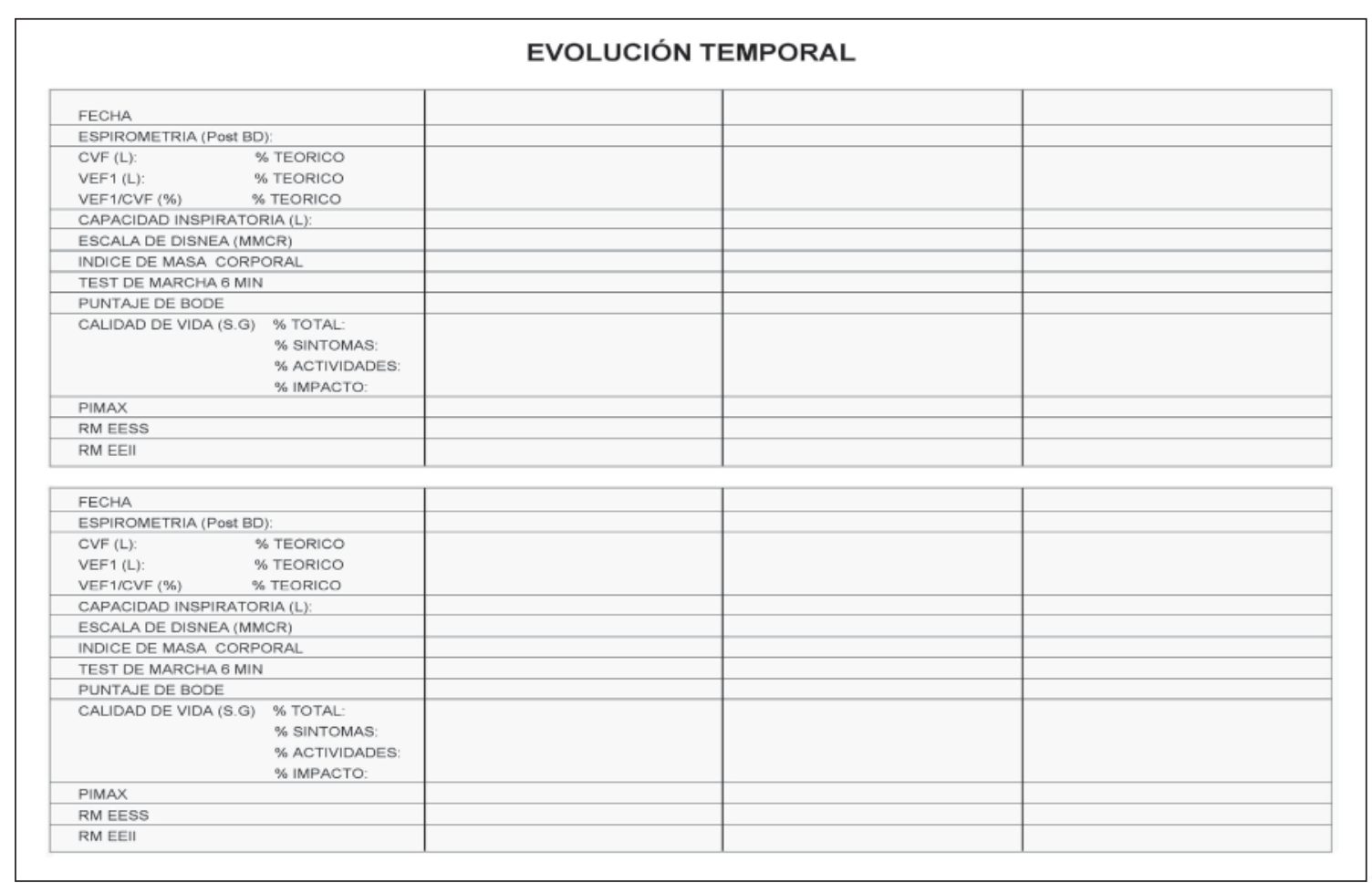

Figura 1. Ficha clínica de recolección de información de los pacientes con enfermedad pulmonar obstructiva crónica que se incorporan a un programa de rehabilitación respiratoria. 
realizar la evaluación de los pacientes, una sala de entrenamiento amplia y ventilada y un pasillo para realizar la prueba de caminata de 6 minutos $^{41}$. No obstante, se han implementado programas de rehabilitación respiratoria ambulatorios en el domicilio del paciente, con buenos resultados clínicos ${ }^{54}$.

En cuanto al equipamiento necesario para realizarla un programa de rehabilitación respiratoria, se requiere el siguiente equipo:

\begin{tabular}{|l|l|}
\hline Mínimo & Opcionales \\
\hline Oxímetro de pulso & Pesa \\
Monitor de frecuencia cardíaca & Espirómetro \\
Esfigmomanómetro & Glucómetro \\
Treadmill y/o cicloergómetro & \\
Mancuernas & \\
Bandas elásticas & \\
Podómetros y/o cuentapasos & \\
Escabel/Step & \\
\hline Equipo de emergencia: carro de & \\
paro y personal entrenado & \\
\hline
\end{tabular}

\section{Duración y frecuencia del programa de rehabilitación respiratoria}

Se recomienda que el programa de rehabilitación respiratoria tenga una duración de 6 a 12 semanas, con al menos tres sesiones semanales supervisadas y cada sesión de 3 a 4 horas de duración ${ }^{3}$. En los pacientes con compromiso funcional grave, puede ser necesario prolongar el entrenamiento hasta los 6 meses.

\section{Donde realizar los programas de rehabilitación respiratoria}

Se ha demostrado que la rehabilitación respiratoria es una intervención efectiva en pacientes con EPOC hospitalizados y ambulatorios, manejados en el hospital y en el hogar. Por otro lado, una revisión sistemática de la literatura realizada el año 2010 concluye que la rehabilitación respiratoria automonitoreada y efectuada en el domicilio, puede ser una alternativa a los programas extrahospitalarios, lo que facilitaría su implementación y la accesibilidad de los pacientes. La efectividad del programa es independiente del lugar en que se lleva a cabo y depende fundamentalmente de la estructura del programa $a^{1,9,55}$.

\section{Duración y prolongación del efecto de la rehabilitación pulmonar}

Los beneficios de la rehabilitación respiratoria, en programas de 6-12 semanas, se mantienen durante 1 a 2 años $^{9}$ y en programas de más larga duración ( $>12$ semanas) los beneficios perduran más tiempo. Las técnicas de mantenimiento tie- nen un leve efecto sobre los beneficios a largo plazo'.

Es recomendable mantener durante el mayor tiempo posible los efectos favorables de la rehabilitación respiratoria ${ }^{1}$, para ello se sugiere realizar: 1) Programas sucesivos de rehabilitación pulmonar; 2) Programas de rehabilitación respiratoria efectivos; 3) Emplear herramientas para incentivar la actividad física luego de finalizada la rehabilitación pulmonar; 4) Prolongar la duración de los programas de rehabilitación pulmonar.

\section{Evaluación de la eficacia de un programa de rehabilitación respiratoria}

Cuando se organiza un programa de rehabilitación respiratoria, es importante plantear una estrategia de estudio y control, por tanto, el programa debe constar de: 1) Evaluación inicial; 2) Período de intervención (RR) y 3) Evaluación final. Los parámetros que serán evaluados al ingreso y al término del programa son: 1) Magnitud de la disnea, fatigabilidad, depresión/ansiedad; 2) Calidad de vida relacionada con la salud (CVRS); 3) Capacidad de realizar actividad física y esfuerzo; 4) Costos y 5) Supervivencia.

\section{Grado de recomendación}

Todos los pacientes con EPOC, excepto aquellos con criterios de exclusión, se benefician de los programas de rehabilitación respiratoria con mejoría significativa de los síntomas respiratorios, capacidad de realizar ejercicio y calidad de vida (calidad de la evidencia A, calidad de la recomendación fuerte).

\section{Conclusiones}

Los estudios clínicos controlados y las evaluaciones sistemáticas han demostrado fehacientemente los beneficios clínicos de los programas de rehabilitación respiratoria en pacientes con enfermedad pulmonar obstructiva crónica. La aplicación de un programa de rehabilitación respiratoria debe ser individualizada y adecuada a cada paciente. El paciente debe ser evaluado inicialmente desde el punto de vista clínico, radiológico, de función pulmonar, de capacidad de esfuerzo y de calidad de vida relacionada con la salud. De esta forma, se puede establecer el grado de limitación o incapacidad física del paciente, y se podrá diseñar un programa de rehabilitación personalizado centrado en las necesidades del paciente. Del mismo modo, es conveniente repetir las mediciones al finalizar el programa, con el objetivo de poder evaluar los resultados del 
mismo. La rehabilitación respiratoria puede realizarse en el hospital, en los servicios de atención primaria y/o en el domicilio del paciente; las tres modalidades han demostrado ser efectivas.

\section{Bibliografía}

1.- NICI L, DONNER C, WOUTERS E, ZUWALLACK R, AMBROSINO N, BOURBEAU J, et al. ATS/ERS Pulmonary Rehabilitation Writing Committee. American Thoracic Society/ European Respiratory Society statement on pulmonary rehabilitation. Am J Respir Crit Care Med 2006; 173: 1390-413.

2.- MURRAY C J, LÓPEZ A D. Alternative projections of mortality and disability by cause 1990-2020: Global Burden of Disease Study. Lancet 1997; 349: 1498-504.

3.- TROOSTERS T, CASABURI R, GOSSELINK R, DECRAMER M. Pulmonary rehabilitation in chronic obstructive pulmonary disease. Am J Respir Crit Care Med 2005; 172: 19-38.

4.- FOSTER S, THOMAS H M. $3^{\text {rd }}$. Pulmonary rehabilitation in lung disease other than chronic obstructive pulmonary disease. Am Rev Respir Dis 1990; 141: 601-4.

5.- NISHIYAMA O, KONDOH Y, KIMURA T, KATO K, KATAOKA K, OGAWA T, et al. Effects of pulmonary rehabilitation in patients with idiopathic pulmonary fibrosis. Respirology 2008; 13: 394-9.

6.- SALHI B, TROOSTERS T, BEHAEGEL M, JOOS G, DEROM E. Effects of pulmonary rehabilitation in patients with restrictive lung diseases. Chest 2010; 137: 273-9.

7.- FABBRI L M, LUPPI F, BEGHÉ B, RABE K F. Complex chronic comorbidities of COPD. Eur Respir J 2008; 31: 204-12.

8.- GROUP GDG. Managing stable COPD. Thorax 2004; 59 (Suppl I): 1-232.

9.- RIES A L, BAULDOFF G S, CARLIN B W, CASABURI R, EMERY C F, MAHLER D A, et al. Pulmonary rehabilitation: Joint ACCP/AACVPR Evidence-Based Clinical Practice Guidelines. Chest 2007; 131 (5 Suppl): 4S-42S.

10.- SEPAR-ALAT. Guía de práctica clínica de diagnóstico y tratamiento de la Enfermedad Pulmonar Obstructiva Crónica. 2009. www.separ.es.

11.- LACASSE Y, GOLDSTEIN R, LASSERSON T J, MARTIN S. Pulmonary rehabilitation for chronic obstructive pulmonary disease. Cochrane Database Syst Rev 2006; 4: CD003793.

12.- MARÍN K, LAUDE R, MORALES C. Entrenamiento físico y educación como parte de la rehabilitación pulmonar en pacientes con EPOC. Rev Chil Enf Respir 2008; 24: 286-90.

13.- GLOBAL INITIATIVE FOR CHRONIC OBSTRUCTIVE LUNG DISEASE. Global strategy for the diagnosis, management, and prevention of chronic obstructive lung disease (Updated 2009). [Accessed 22 May 2010.]
Available from URL: http//www.goldcopd.com/ GuidelinesResources.

14.- SÍVORI M, BENZO R, RHODIUS E, JOLLY E, BOIM $\mathrm{C}$, SAADIA M, et al. Consenso argentino de rehabilitación respiratoria. Medicina (B Aires) 2004; 64: 357-67.

15.- LACASSE Y, WONG E, GUYATT G H, KING D, COOK D J, GOLDSTEIN R S. Meta-analysis of respiratory rehabilitation in chronic obstructive pulmonary disease. Lancet 1996; 348: 1115-9.

16.- GRIFFITHS T L, BURR M L, CAMPBELL I A, LEWIS-JENKINS V, MULLINS J, SHIELS K, et al. Results at 1 year of outpatient multidisciplinary pulmonary rehabilitation: a randomised controlled trial. Lancet 2000; 355: 362-8.

17.- PUHAN M A, SCHARPLATZ M, TROOSTERS T, STEURER J. Respiratory rehabilitation after acute exacerbation of COPD may reduce risk for readmission and mortality -a systematic review. Respir Res 2005; 6 : 54.

18.- MAN W D, POLKEY M I, DONALDSON N, GRAY B J, MOXHAM J. Community pulmonary rehabilitation after hospitalization for acute exacerbations of chronic obstructive pulmonary disease: randomised controlled study. Br Med J 2004; 329: 1209.

19.- STAV D, RAZ M, SHPIRER I. Three years of pulmonary rehabilitation: inhibit the decline in airflow obstruction, improves exercise endurance time, and body-mass index, in chronic obstructive pulmonary disease. BMC Pulm Med 2009; 9: 26.

20.- COTE C G, CELLI B R. Pulmonary rehabilitation and the BODE index in COPD. Eur Respir J 2005; 26: 6306.

21.- BERRY M J, REJESKI W J, ADAIR N E, ZACCARO D. Exercise rehabilitation and chronic obstructive pulmonary disease stage. Am J Respir Crit Care Med 1999; 160: 1248-53.

22.- CHAVANNES N, VOLLENBERG J J, VAN SCHAYCK C P, WOUTERS E F. Effects of physical activity in mild to moderate COPD: a systematic review. $\mathrm{Br} \mathrm{J}$ Gen Pract 2002; 52: 574-8.

23.- BRITISH THORACIC SOCIETY Standards of Care Subcommittee on Pulmonary Rehabilitation. Pulmonary rehabilitation. Thorax 2001; 56: 827-34.

24.- SABIT R, GRIFFITHS T L, WATKINS A J, EVANS W, BOLTON C E, SHALE D J, et al. Predictors of poor attendance at an outpatient pulmonary rehabilitation programme. Respir Med 2008; 102: 819-24.

25.- HAAVE E, SKUMLIEN S, HYLAND M E. Gender considerations in pulmonary rehabilitation. J Cardiopulm Rehabil Prev 2008; 28: 215-9.

26.- DI MEO F, PEDONE C, LUBICH S, PIZZOLI C, TRABALLESI M, INCALZI R A. Age does not hamper the response to pulmonary rehabilitation of COPD patients. Age Ageing 2008; 37: 530-5.

27.- YOUNG P, DEWSE M, FERGUSSON W, KOLBE J. Respiratory rehabilitation in chronic obstructive pulmo- 
nary disease: predictors of nonadherence. Eur Respir J 1999; 13: 855-9.

28.- ANTHONISEN N R, WRIGHT E C, HODGKIN J E. Prognosis in chronic obstructive pulmonary disease. Am Rev Respir Dis 1986; 133: 14-20.

29.- JONES P W. Health status measurement in chronic obstructive pulmonary disease. Thorax 2001; 56: 880-7.

30.- SPENCER L M, ALISON J A, MCKEOUGH Z J. Six-minute walk test as an outcome measure: are two six-minute walk tests necessary immediately after pulmonary rehabilitation and at three-month follow-up? Am J Phys Med Rehabil 2008; 87: 224-8.

31.- JENKINS S, CECINS N M. Six-minute walk test in pulmonary rehabilitation: do all patients need a practice test? Respirology 2010; 15: 1192-6.

32.- ATS Committee on Proficiency Standards for Clinical Pulmonary Function Laboratories. ATS statement: guidelines for the six-minute walk test. Am J Respir Crit Care Med 2002;166:111-7.

33.- PUHAN M A, MADOR M J, HELD U, et al. Interpretation of treatment changes in six-minute walk distance in patients with COPD. Eur Respir J 2008; 32: 637-43.

34.- HOLLAND A E, HILL C J, RASEKABA T, LEE A, NAUGHTON M T, MCDONALD C F. Updating the minimal important difference for six-minute walk distance in patients with chronic obstructive pulmonary disease. Arch Phys Med Rehabil 2010; 91: 221-5.

35.- REDELMEIER D A, BAYOUMI A M, GOLDSTEIN R S, GUYATT G H. Interpreting small differences in functional status: the six minute walk test in chronic lung disease patients. Am J Respir Crit Care Med 1997; 155: 1278-82.

36.- SINGH S J, MORGAN M D, SCOTT S, WALTERS D, HARDMAN A E. Development of a shuttle walking test of disability in patients with chronic airways obstruction. Thorax 1992; 47: 1019-24.

37.- SINGH S J, JONES P W, EVANS R, MORGAN M D. Minimum clinically important improvement for the incremental shuttle walking test. Thorax 2008; 63: 775-7.

38.- BORG G A. Psychophysical bases of perceived exertion. Med Sci Sports Exerc 1982; 14: 377-81.

39.- LOISEAU A, DUBREUIL C, PUJET J C. A visual analog scale of exercise dyspnea. Rev Mal Respir 1990; 7 : 39-44.

40.- BESTALL J C, PAUL E A, GARROD R, GARNHAM R, JONES P W, WEDZICHA J A. Usefulness of the Medical Research Council (MRC) dyspnoea scale as a measure of disability in patients with chronic obstructive pulmonary disease. Thorax 1999; 54: 581-6.

41.- JENKINS S, HILL K, CECINS N M. State of the art: how to set up a pulmonary rehabilitation program. Respirology 2010; 15: 1157-73.

42.- JONES P W. Interpreting thresholds for a clinically significant change in health status in asthma and COPD. Eur Respir J 2002; 19: 398-404.

43.- KOPPERS R, VOS P J, BOOT C, FOLGERING H T.
Exercise performance improves in patients with COPD due to respiratory muscle endurance training. Chest 2006; 129: 886-92.

44.- SMITH K, COOK D, GUYATT G H, MADHAVAN J, OXMAN A D. Respiratory muscle training in chronic airflow limitation: a meta-analysis. Am Rev Resp Dis 1992; 145: 533-9.

45.- CELLI B R, RASSULO J, MAKE B J. Dyssynchronous breathing during arm but not leg exercise in patients with chronic airflow obstruction. N Engl J Med 1986; 314: 1485-90.

46.- CELLI B R, COTE C G, MARÍN J M, CASANOVA C, MONTES DE OCA M, MÉNDEZ R A, et al. The bodymass index, airflow obstruction, dyspnea, and exercise capacity index in chronic obstructive pulmonary disease. N Engl J Med 2004; 350: 1005-12.

47.- GÜELL R, RESQUETI V, SANGENIS M, MORANTE F, MARTORELL B, CASAN P, et al. Impact of pulmonary rehabilitation on psychosocial morbidity in patients with severe COPD. Chest 2006; 129: 899-904.

48.- TOSHIMA M T, BLUMBERG E, RIES A L, KAPLAN $\mathrm{R}$ M. Does rehabilitation reduce depression in patients with chronic obstructive pulmonary disease? J Cardiopulm Rehabil 1992; 12: 261-9.

49.- ZIGMOND A S, SNAITH R P. The hospital anxiety and depression scale. Acta Psychiatr Scand 1983; 67: 361-70.

50.- STEINER M C, BARTON R L, SINGH S J, MORGAN $M$ D. Nutritional enhancement of exercise performance in chronic obstructive pulmonary disease: a randomised controlled trial. Thorax 2003; 58: 745-51.

51.- FERREIRA I M, BROOKS D, LACASSE Y, GOLDSTEIN R S. Nutritional support for individuals with COPD: a meta-analysis. Chest 2000; 117: 672-8.

52.- FERREIRA I, BROOKS D, LACASSE Y, GOLDSTEIN R. Nutritional intervention in COPD: a systematic overview. Chest 2001; 119: 353-63.

53.- GLAAB T, VOGELMEIER C, BUHL R. Outcome measures in chronic obstructive pulmonary disease (COPD): strengths and limitations. Respir Res 2010; 11: 79.

54.- GÜELL M R, DE LUCAS P, GÁLDIZ J B, MONTEMAYOR T, RODRÍGUEZ GONZÁLEZ-MORO J M, GOROSTIZA A, ORTEGA F, BELLÓN J M, GUYATT G. Home $v s$ hospital-based pulmonary rehabilitation for patients with chronic obstructive pulmonary disease: a Spanish multicenter trial. Arch Bronconeumol 2008; 44: 512-8.

55.- VIEIRA D S, MALTAIS F, BOURBEAU J. Homebased pulmonary rehabilitation in chronic obstructive pulmonary disease patients. Curr Opin Pulm Med 2010; 16: 134-43.

Correspondencia a:

Dra. Juana Pavié G.

Servicio de Medicina, Hospital San Martín.

Quillota, V Región.

E-mail: juanipavie@gmail.com 